\title{
Research on Decorative Style Illustration Design
}

\author{
Li suo ${ }^{1}$, Yingwei Li \\ ${ }^{1}$ JingDeZhen Ceramic Institute, JiangXi, JingDeZhen, 333403
}

Keywords: decorative style; illustration design

\begin{abstract}
Illustrations, as an important form of art design, have a wide range of applications in modern society. The theme of illustration is more prominent with a stronger visual impact, can give people unlimited imagination, can make life full of fun, and can improve people's visual experience. The decorative style in the illustration design also presents a variety of features, selects the appropriate style according to individual needs and the environment, and ultimately achieves the perfect expression of art to bring people a new visual experience.
\end{abstract}

\section{Introduction}

As an ancient and novel art, illustration is an important part of the visual language. It can combine art design with modern technology, and has more artistic appeal and timeliness. With the development of computer technology, the style of illustrations is more diversified, and modern decorative styles are continuously enriched with the development of technology, effectively satisfying people's individual needs [1].

As an art expression, illustration is an important visual expression in modern design. It can express beauty through intuitive phenomena and reflect real life. With the rapid development of society, the application of illustrations has become more and more extensive, and the modern decorative style has become increasingly diversified. Illustrations have been applied to various fields in society, such as general business activities, cultural activities, and film and television industries. The emergence of illustration design enriches people's lives, opens up people's horizons, brings beauty to people's feelings, and also expands people's thinking space, enables people to observe life from a new perspective, think about life, and become people's lives. important parts of. With the rapid development of the market economy, the meaning and scope of illustrations have become more and more extensive. The illustration design style is colorful and no longer limited to a certain style. In the specific design, some designers used a variety of materials to express the tension of the pattern, no longer confined to a single material, thus showing a unique artistic effect, so that the illustration art has been rapidly developed, greatly expanding the illustration The space for expression. Illustrations have a strong vitality in modern society, and with the constant progress of technology, its style is more and more diverse.

\section{The Status Quo of the Illustration Design at Home and Abroad}

The development of modern commercial illustrations is generally speaking, the illustrations of service nature are enriched and developed in terms of content, and the artistic expression means and style of illustrations have also undergone tremendous changes. Illustrations have also been extended in various media. Commercial illustrations have gradually gained the attention of the masses and many media and realized their cultural values.

In the modern design field, the illustration design has been extended from a single print medium to new media such as film and television, animation, mascots, apparel, software, and construction, and has a very broad development prospect. Modern illustrations began to gradually shift from the traditional painting techniques and expression methods to the digital age. This feature makes us feel the unprecedented appeal of illustrations. With the continuous extension of the media of modern illustrations, the carrier of illustration has also undergone tremendous changes. The commercial illustrator is the inevitable product of great changes in the illustrator industry [2]. 
Modern illustration design has also involved various application media in Europe, America, Japan, France and other countries and has gained new development and artistic achievements. Especially in the field of network and digital technology, constantly expanding our horizons has enriched our visual experience and has further opened up our minds. Commercial illustrations have played a huge role in promoting economic development. The artistic style of commercial illustrations has been correspondingly developed and improved with the diversification of different application media. The application of commercial illustrations combined with the application of various media has also been continuously enriched, resulting in the unprecedented development of modern illustration art.

\section{Visual Language in Contemporary Commercial Illustration Decoration}

Some of the styling features of contemporary commercial illustration decoration not only inherited traditional language expressions, such as exaggeration and planarization, but also used some new expression techniques that reflected the characteristics of the times, fully embodying the rich and varied features of visual language features.

In contemporary commercial illustrations with decorative styles, no matter how complex the pictures are, the specific edges of the shapes represented by each specific thing are clear and distinct. This, to a certain extent, inherits the characteristics exhibited by the motif in the traditional decorative style. Such as: "Decorative Art Movement" reflected in the simple and distinct geometry. In today's society, with the development of technology, the powerful function of drawing software makes all kinds of things more clear and prominent. The vectorization of the contour is basically determined by the vector technique. Powerful vector technology makes the previous vague feelings no longer exist, the various features of the things are clear, the form of the picture is stronger, fully embodies the decoration style of commercial illustration in the new era [3].

In the same picture, the repeated use of one or more elements is also extracted from the traditional decorative style. In today's digital era, with the help of various technologies, this technique of expression has also been applied to the extreme. In a picture, using an element repeatedly, you can use a certain cell as a starting point, and make the rhythm of the picture clearer and the color more prominent by copying, adjusting density, reorganizing, enlarging, reducing, changing colors, and so on. In addition, using some elements to form a regular pattern, filling in some irregular objects will achieve a very good effect. For example, Takashi Murakami’s illustrated work "Flower" uses repeated techniques for background design.

In terms of visual effects, color plays a leading role, and the same color plays an important role in visual communication. Although the symbolic meaning of color is different due to cultural differences in different regions, the color still has full charm. When people appreciate a work, the direct stimulation of color to the human mind will directly affect people's perception of art illustrations and play a specific psychological effect.

In modern commercial illustration decoration, most of the use of color is arbitrary, and there will be no situation in traditional painting. For example, the emphasis on the harmony of the picture colors, the limited use of the inherent color of the object itself, and the full consideration of the external environment Factors such as the impact. The use of color is more dependent on the individual's personal aesthetic habits and personality, guided by the intuition of the creator. In the aspect of the decoration of contemporary commercial illustrations, the use of color mainly has these characteristics: subjectivity, uncertainty, and non-predictability, and the expression of color use is entirely a manifestation of subjective consciousness. To a certain extent, the artist's life status and personality characteristics can be clearly understood from the works. Therefore, some art and painting designers prefer to use some elegant colors, and some art and painting designers prefer bright and crisp colors.

\section{The Performance Style of Contemporary Commercial Illustrations in the Decorative Style}

The decorative style of commercial illustration works is more about the use of formal beauty by 
creators, such as the contrastive use of cold and warm colors on tones, the processing of three-dimensional space and plane construction, the prominent contrast between vector graphics and texture, and the synthesis of straight lines and curves. Use, and so on, through the use of these contrasting effects to create a rich and profound picture. Contemporary young business illustrators, who love to extract nutrients from historical and cultural traditions, extract decorative elements from traditional artistic decorations and apply them to today's multi-element culture, will form a very special artistic language. The decorative arts in the national tradition are the treasures of the national art, fully embodying the rich heritage and cultural connotations of the national art. Many of today's finest commercial illustrators have refined the elements they need from traditional ethnic decorations, thus forming a unique personal art language.

The Japanese illustrator Katoki is a typical painter who makes full use of the decorative elements in traditional culture. Her works not only have a strong oriental traditional decorative charm, but also have distinctive personal characteristics. Most of Kato's works are famous for their gorgeous and classic styles. In her works, she fully reveals a delicate temperament unique to women. At the same time, she also has some unrestrained feelings and gives people a great imagination. Her favorite colors, such as yellow, black, red, and purple, are widely used in her works. Her illustrations are mostly women's themes, combined with Japanese comics, costumes, various fairy tales, and religious elements to achieve certain artistic realms. Her style is even known as "Yazzi of the Oriental Ratio."

The use of contemporary business illustrations in the decoration of geometric figures arranged, combined with simple graphics such as points, lines, circles, triangles, squares, etc. has some intuitive visual experience. Different graphic combinations and combinations of different shades can create different visual effects in space. Different topics can be achieved in the presentation of topics. Points, lines, and faces also have a very special role in performance.

The works of contemporary Japanese master Tanaka Ikegami are unique to the use of visual elements. He has a piece of "Japanese Dance" with the "face" as the object. He fully uses the geometrical dichotomy, and at the same time uses the expression of the semicircle to break the expression of conventional dance and give people full imagination [4].

In the contemporary commercial illustration decoration, the illustrator artists have incorporated cartoons, animations, cartoons and other elements into the illustrations, changing the traditional animation impressions and forming a unique pop style.

Murakami Takashi is an influential Japanese contemporary artist. His works have both oriental traditions and western civilizations. The contrast between elegant and popular elements makes his works not only ornamental but also entertaining. One of Murakami's works is to symbolize Mickey Mouse. Another Stephen Kelleher's work is also a combination of a large number of cartoon elements, through a simple process, making it exist as a graphic symbol.

\section{The Application of Illustration in Decoration}

Illustrations have a strong ability to infect, can intuitively and vividly pass out their information and images, and thus arouse people's attention. In recent years illustrations have been rapidly developed in various industries, such as games and animation industries. Illustrations also have a wide range of applications in modern decoration. It enriches people's lives and can give people extraordinary visual experience. In the common furniture industry, using illustrations can beautify people's lives and add fun to people's lives. In the decoration of the furniture, more illustrations are used for the decoration, and the illustrations can be used to decorate the furniture so that the furniture can achieve more beautiful artistic effects. For example, it is possible to decorate the surface of the furniture so that the furniture exhibits a unique aesthetic feeling; it is also possible to make use of the linear decoration so that the surface of the furniture exhibits a convex-concave effect and enriches the three-dimensional effect of the furniture. In the furniture decoration is more used in the pattern, this method has been widely used in ancient China, such as the use of more auspicious animals, plants and other motifs in Chinese classical furniture to decorate, express The research on the interior style of the interior walls of people's expectations of beautifying life, local 
customs, and unique aesthetic tastes

With the continuous improvement of people's quality of life, there are also relatively high demands on the living environment. In particular, with the development of people's personality and the growing demand for fashion, the wall has become an important part of modern architectural decoration. The ordinary wall surface has been difficult to meet people's needs. People often perform hand paintings on the walls and effectively enrich the interior architectural space. Hand painting is an important part of the wall decoration, which enriches the language of interior decoration and plays an important role in the design of the home environment. Currently the theme of the wall illustration design is rich, the style is also very diversified, there is a stylish Scandinavian style, there are retro Chinese style, there are cartoon style full of childlike fun, natural quiet rural style and so on. Among them, the selection of Chinese style painting patterns is mainly the pattern of our country's traditional patterns, or the use of Chinese traditional ink painting techniques to represent the patterns of flowers and birds. Nordic style wall patterns are mainly composed of abstract graphics, which are more colorful or simple black and white in color. The cartoon-style wall is mainly used in children's rooms. The wall is full of colorful cartoon characters or animals. The style and illustration of the wall can be determined according to the needs of the design, closely related to the owner's aesthetic taste, personality, and the decorative style and function of the architectural space [5].

Cartoon patterns are widely used in wall illustration design. Cartoon patterns can meet the needs of children's psychology. When choosing a pattern, you should choose a cartoon image that children are familiar with and like, such as the common Pink Pig, Snow White, Winnie the Pooh and the Tigger, combined with small animals and balloons of various colors, skyscrapers, etc. The entire pattern looks colorful. A cartoon sun pattern can be designed on the roof wall to make the room seem full of sunlight. The choice of wall patterns should be based on the children's psychological needs, organize a variety of cartoon shapes and into the wall scene, so that the content is rich and interesting. In practice, children are found to have unique aesthetic needs. They generally like some colorful designs. These patterns often give them a strong visual appeal and impact that can attract their attention. In the design of the pattern, attention should be paid to the selection and application of colors. In general, bright colors such as solid colors and primary colors should be selected. You can use the blue sky and white clouds and green grass as the background. This kind of color can give a sense of vitality, a symbol of vitality, and can arouse children's interest. In the pattern of expression, according to the children's thinking characteristics, choose a flat means of expression, so that children can easily understand and accept. The combination of wall illustrations and other play facilities in the children's room can effectively integrate the entire space and create a playful fairy tale world and space.

\section{Conclusion}

As an important visual art, illustration design enriches people's lives and has been widely used in modern decoration. The modern decoration style not only includes the traditional art style, but also develops a new illustration style. For example, its fashionable style is more intense and can meet the development needs of modern society. With the development of many meta-cultures, illustrations such as cartoons have also been widely recognized and welcomed, showing unique charm.

\section{References}

[1] Wu Yizhi. Commercial illustration design in the context of cultural globalization [J]. Packaging Engineering. 2007 (07).12

[2] Li Guang. Li Guang Illustration Design Works [J]. Decoration. 2007 (12).33

[3] Xia Ping. The Present Situation and Trend of Illustration Design Teaching [J]. Art Grand View. 2009 (03).67

[4] Chen Tao. Research and Exploration of Illustration Design Teaching [J]. Reading and Writing 
(educational journal). 2010 (03).55

[5] Liao Kejun. Exploration of Modern Illustration Design Teaching [J]. Popular Literature. 2010 (24).124 